\title{
On the Adjacency Matrix and Neighborhood Associated with Zero-divisor Graph for Direct Product of Finite Commutative Rings
}

\author{
Kuntala Patra \\ Department of Mathematics \\ Gauhati University \\ Guwahati - 781014, India
}

\author{
Priyanka Pratim Baruah \\ Department of Mathematics \\ Girijananda Chowdhury Institute of Management and \\ Technology \\ Guwahati - 781017, India
}

\begin{abstract}
The main purpose of this paper is to study the zero-divisor graph for direct product of finite commutative rings. In our present investigation we discuss the zero-divisor graphs for the following direct products: direct product of the ring of integers under addition and multiplication modulo $p$ and the ring of integers under addition and multiplication modulo $p^{2}$ for a prime number $p$, direct product of the ring of integers under addition and multiplication modulo $p$ and the ring of integers under addition and multiplication modulo $2 p$ for an odd prime number $p$ and direct product of the ring of integers under addition and multiplication modulo $p$ and the ring of integers under addition and multiplication modulo $p^{2}-2$ for that odd prime $p$ for which $p^{2}-2$ is a prime number. The aim of this paper is to give some new ideas about the neighborhood, the neighborhood number and the adjacency matrix corresponding to zero-divisor graphs for the above mentioned direct products. Finally, we prove some results of annihilators on zerodivisor graph for direct product of $A$ and $B$ for any two commutative rings $A$ and $B$ with unity
\end{abstract}

Keywords: Zero-divisor, Commutative ring, Adjacency matrix, Neighborhood, Zero-divisor graph, Annihilator.

AMS Classification (2010): 05Cxx; 05C25; 05C50.

\section{INTRODUCTION}

The idea of zero-divisor graph of a commutative ring was first introduced by I. Beck [2] in 1988. D. F. Anderson and P.S. Livinsgston [1] redefined the concept of zero-divisor graph in 1999. F. R. DeMeyer, T. Mckenzie and K. Schneider [3] extended the concept of zero-divisor graph for commutative semi-group in 2002. The notion of zerodivisor graph had been extended for non-commutative rings by S. P. Redmond [9] in 2002. Recently, P. Sharma, A. Sharma and R. K. Vats [10] have discussed the neighborhood set, the neighborhood number and the adjacency matrix of zero-divisor graphs for the rings $Z_{p} \times Z_{p}$ and $Z_{p}[i] \times Z_{p}[i]$, where $p$ is a prime number.

In this paper $R_{l}$ denotes the finite commutative ring such that $R_{l}=Z_{p} \times Z_{p^{2}}$ ( $p$ is a prime number), $R_{2}$ denotes the finite commutative ring such that $R_{2}=Z_{p} \times Z_{2 p}$ ( $p$ is an odd prime number) and $R_{3}$ denotes the finite commutative ring such that $R_{3}=Z_{p} \times Z_{p^{2}-2}$ ( for that odd prime $p$ for which $p^{2}-2$ is a prime number). Let $R$ be a commutative ring with unity and $\mathrm{Z}(R)$ be the set of zero-divisors of $R$; that is $\mathrm{Z}(R)=\left\{x \in R: x y=0\right.$ or $y x=0$ for some $y \in R^{*}=R-$ $\{0\}\}$.Then zero-divisor graph of $R$ is an undirected graph $\Gamma(R)$ with vertex set $\mathrm{Z}(R)^{*}=\mathrm{Z}(R)-\{0\}$ such that distinct vertices $x$ and $y$ of $Z(R)^{*}$ are adjacent if and only if $x y=0$. The neighborhood (or open neighborhood) $N_{G}(v)$ of a vertex $v$ of a graph $G$ is the set of vertices adjacent to $v$. The closed neighborhood $N_{G}[v]$ of a vertex $v$ is the set $N_{G}(v) \cup\{v\}$. For a set $S$ of vertices, the neighborhood of $S$ is the union of the neighborhoods of the vertices and so it is the set of all vertices adjacent to at least one member of $S$. For a graph $G$ with vertex set $V$, the union of the neighborhoods of all the vertices is neighborhood of $V$ and it is denoted by $N_{G}(V)$. The neighborhood number $n_{G}(V)$ is the cardinality of $N_{G}(V)$. If the graph $G$ with vertex set $V$ is connected, then $N_{G}(V)$ is the vertex set $V$ and the cardinality of $N_{G}(V)$ is equal to the cardinality of $V$. If $\Gamma(R)$ is the zero-divisor graph of a commutative ring $R$ with vertex set $\mathrm{Z}(R)^{*}$ and since zerodivisor graph is always connected [1], we have $N_{\Gamma(R)}\left(\mathrm{Z}(R)^{*}\right)=$ $\mathrm{Z}(R)^{*}$ and $\left.\left|N_{\Gamma(R)}\left(\mathrm{Z}(R)^{*}\right)\right|=\mid \mathrm{Z}(R)^{*}\right) \mid$. Throughout this paper $\Delta(G)$ denotes the maximum degree of a graph $G$ and $\delta(G)$ denotes the minimum degree of a graph $G$. The adjacency matrix corresponding to zero-divisor graph $G$ is defined as $A=\left[a_{i j}\right]$, where $a_{i j}=1$, if $v_{i} v_{j}=0$ for any vertex $v_{i}$ and $v_{j}$ of $G$ and $a_{i j}=0$, otherwise.

In this paper, we construct zero-divisor graphs for the rings $R_{l} R_{2}$ and $R_{3}$. We obtain the neighborhood and the adjacency matrices corresponding to zero-divisor graphs of $R_{I}, R_{2}$ and $R_{3}$. Some properties of adjacency matrices are also obtained. We prove some theorems related to neighborhood and adjacency matrices corresponding to zero-divisor graphs of $R_{1} R_{2}$ and $R_{3}$. Finally, we prove some results of annihilators on zero-divisor graph of $A \times B$, for any two commutative rings $A$ and $B$ with unity. 


\section{CONSTRUCTION OF ZERO -DIVISOR GRAPH FOR $\boldsymbol{R}_{\boldsymbol{l}}=Z_{p} \times Z_{p^{2}}(p$ IS A PRIME NUMBER):}

First, we construct the zero-divisor graph for the ring $R_{I}=Z_{p} \times Z_{p^{2}}(p$ is a prime number) and analyze the graph. We start with the cases $p=2$ and $p=3$ and then generalize the cases.

Case 1: When $p=2$ we have $R_{1}=Z_{2} \times Z_{4}$

The ring $R_{I}$ has 5 non-zero zero-divisors. In this case $V=$ $\mathrm{Z}\left(R_{l}\right)^{*}=\{(1,0),(0,1),(0,2),(0,3),(1,2)\}$ and the zero-divisor graph $G=\Gamma\left(R_{l}\right)$ is given by:

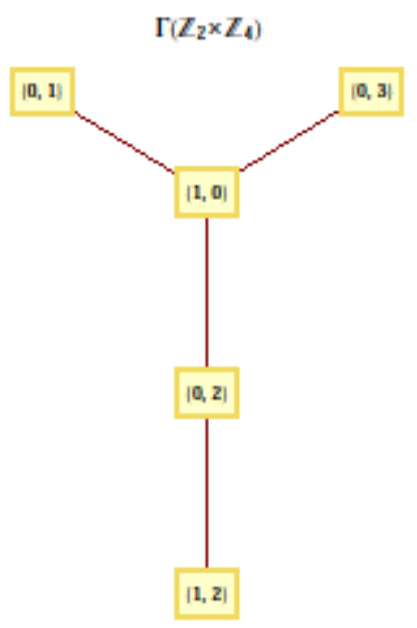

Fig: 1

The closed neighborhoods of the vertices are $N_{G}[(1,0)]=\{(1,0),(0,1),(0,2),(0,3)\}, N_{G}[(0,1)]=\{(1,0),(0,1)\}$, $N_{G}[(0,2)]=\{(1,0),(1,2),(0,2)\}, N_{G}[(0,3)]=\{(1,0),(0,3)\}$ and $N_{G}[(1,2)]=\{(0,2),(1,2)\}$. The neighborhood of $V$ is given by $N_{G}(V)=\{(1,0),(0,1),(0,2),(0,3),(1,2)\}$. The maximum degree is $\Delta(G)=3$ and minimum degree is $\delta(G)=1$. The adjacency matrix for the zero-divisor graph of $R_{I}=Z_{2} \times Z_{4}$ is $M_{I}=\left[\begin{array}{ccc}0 & A_{1 \times 3} & 0 \\ A_{3 \times 1}^{T} & O_{3 \times 3} & B_{3 \times 1} \\ 0 & B_{1 \times 3}^{T} & 0\end{array}\right]_{5 \times 5}$ where, $A_{1 \times 3}=\left[\begin{array}{lll}1 & 1 & 1\end{array}\right]$, $B_{3 \times 1}=\left[\begin{array}{l}0 \\ 1 \\ 0\end{array}\right], A_{3 \times 1}^{T}$ is the transpose of $A_{1 \times 3}, B_{1 \times 3}^{T}$ is the transpose of $B_{3 \times 1}$ and $O_{3 \times 3}$ is the zero matrix.

\section{Properties of adjacency matrix $M_{1}$ :}

(i) The determinant of the adjacency matrix $M_{l}$ corresponding to $G=\Gamma\left(R_{l}\right)$ is 0 .

(ii) The rank of the adjacency matrix $M_{I}$ corresponding to $G=$ $\Gamma\left(R_{l}\right)$ is 2 . (iii) The adjacency matrix $M_{l}$ corresponding to $G=\Gamma\left(R_{l}\right)$ is symmetric and singular.

Case 2: When $p=3$ we have $R_{1}=Z_{3} \times Z_{9}$

The ring $R_{l}$ has 14 non-zero zero-divisors. In this case $V=$ $\mathrm{Z}\left(R_{l}\right)^{*}=\{(1,0),(2,0),(1,3),(1,6),(2,3),(2,6),(0,1),(0,2)$, $(0,3),(0,4),(0,5),(0,6),(0,7),(0,8)\}$ and the zero-divisor graph $G=\Gamma\left(R_{l}\right)$ is given by:

$\Gamma\left(\mathbb{Z}_{3} \times \mathbb{Z}_{9}\right)$

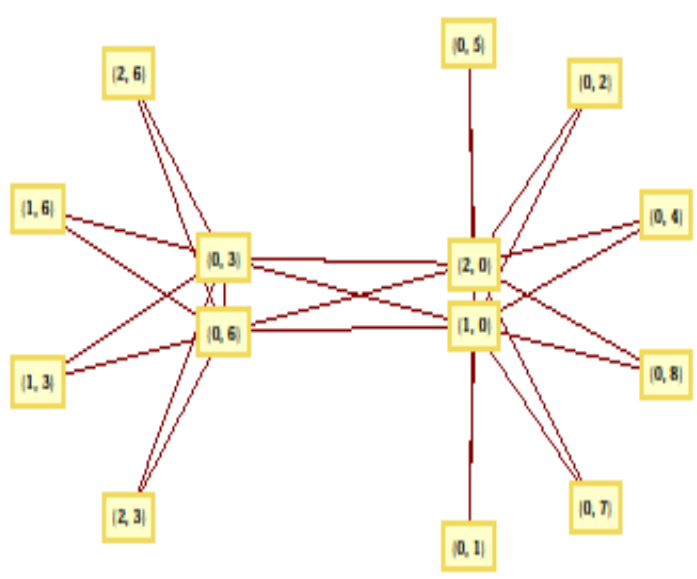

Fig: 2

The closed neighborhoods of the vertices are $N_{G}[(1,0)]=\{(0,1),(0,2),(0,3),(0,4),(0,5),(0,6),(0,7),(0,8)$, $(1,0)\}, N_{G}[(2,0)]=\{(0,1),(0,2),(0,3),(0,4),(0,5),(0,6),(0,7)$, $(0,8),(2,0)\}, N_{G}[(1,3)]=\{(0,3),(0,6),(1,3)\}, N_{G}[(1,6)]=\{(0,3)$, $(0,6),(1,6)\}, N_{G}[(2,3)]=\{(0,3),(0,6),(2,3)\}, N_{G}[(2,6)]=\{(0,3)$, $(0,6),(2,6)\}, N_{G}[(0,1)]=\{(1,0),(2,0),(0,1)\}, N_{G}[(0,2)]=\{(1,0)$, $(2,0),(0,2)\}, N_{G}[(0,3)]=\{(1,0),(2,0),(1,3),(1,6),(2,3),(2,6)$, $(0,6),(0,3)\}, N_{G}[(0,4)]=\{(1,0),(2,0),(0,4)\}, N_{G}[(0,5)]=\{(1,0)$, $(2,0),(0,5)\}, N_{G}[(0,6)]=\{(1,0),(2,0),(1,3),(1,6),(2,3),(2,6)$, $(0,3),(0,6)\}, N_{G}[(0,7)]=\{(1,0),(2,0),(0,7)\}, N_{G}[(0,8)]=\{(1,0)$, $(2,0),(0,8)\}$. The neighborhood of $V$ is given by $N_{G}(V)=$ $\{(1,0),(0,2),(1,3),(1,6),(2,3),(2,6),(0,1),(0,2),(0,3),(0,4)$, $(0,5),(0,6),(0,7),(0,8)\}$. The maximum degree is $\Delta(G)=8$ and minimum degree is $\delta(G)=2$. The adjacency matrix for the zero-divisor graph of $R_{1}=Z_{3} \times Z_{9}$ is $M_{1}=$ $\left[\begin{array}{ccc}O_{6 \times 6} & A_{6 \times 5} & B_{6 \times 3} \\ A^{T}{ }_{5 \times 6} & O_{5 \times 5} & C_{5 \times 3} \\ B^{T}{ }_{3 \times 6} & C^{T}{ }_{3 \times 5} & O_{3 \times 3}\end{array}\right]_{14 \times 14}$ where $A_{6 \times 5}=\left[\begin{array}{lllll}1 & 1 & 1 & 1 & 1 \\ 1 & 1 & 1 & 1 & 1 \\ 0 & 0 & 1 & 0 & 0 \\ 0 & 0 & 1 & 0 & 0 \\ 0 & 0 & 1 & 0 & 0 \\ 0 & 0 & 1 & 0 & 0\end{array}\right]$, $B_{6 \times 3}=\left[\begin{array}{ccc}1 & 1 & 1 \\ 1 & 1 & 1 \\ 1 & 0 & 0 \\ 1 & 0 & 0 \\ 1 & 0 & 0 \\ 1 & 0 & 0\end{array}\right], C_{5 \times 3}=\left[\begin{array}{ccc}0 & 0 & 0 \\ 0 & 0 & 0 \\ 1 & 0 & 0 \\ 0 & 0 & 0 \\ 0 & 0 & 0\end{array}\right], O_{6 \times 6}, O_{5 \times 5}, O_{3 \times 3}$ are the zero matrices and $A_{5 \times 6}^{T}, B^{T}{ }_{3 \times 6}, C^{T}{ }_{3 \times 5}$ are the transposes of $A_{6 \times 5}, B_{6 \times 3}, C_{5 \times 3}$ respectively.

Properties of adjacency matrix $M_{l}$ : 
(i) The determinant of the adjacency matrix $M_{l}$ corresponding to $G=\Gamma\left(R_{l}\right)$ is 0 .

(ii) The rank of the adjacency matrix $M_{l}$ corresponding to $G=$ $\Gamma\left(R_{l}\right)$ is 2 .

(iii) The adjacency matrix $M_{l}$ corresponding to $G=\Gamma\left(R_{l}\right)$ is symmetric and singular.

\section{Generalization for $\boldsymbol{R}_{\boldsymbol{I}}=Z_{p} \times Z_{p^{2}}(\boldsymbol{p}$ is a prime number):}

Lemma 2.1: The number of vertices of $G=\Gamma\left(Z_{p^{2}}\right)$ is $p-1$ and $G=\Gamma\left(Z_{p^{2}}\right)$ is $K_{p-1}$, where $p$ is a prime number [4]

Proof: The multiples of $p$ less than $p^{2}$ are $p, 2 p, 3 p$, $(p-1) p$. These multiples of $p$ are the only non-zero zerodivisors of $Z_{p^{2}}$. If $G=\Gamma\left(Z_{p^{2}}\right)$ is the zero-divisor graph of $Z_{p^{2}}$, then the vertices of $G=\Gamma\left(Z_{p^{2}}\right)$ are the non-zero zero-divisors of $Z_{p^{2}}$. So, the vertex set of $G=\Gamma\left(Z_{p^{2}}\right)$ is $\mathrm{Z}\left(Z_{p^{2}}\right)^{*}$ and $p, 2 p, 3 p, \ldots \ldots \ldots,(p-1) p$ are the vertices of $G=\Gamma\left(Z_{p^{2}}\right)$. Hence, the number of vertices of $G=\Gamma\left(Z_{p^{2}}\right)$ is $p-1$. Also, in $G=\Gamma\left(Z_{p^{2}}\right)$, every vertex is adjacent to every other vertex. This gives $G=\Gamma\left(Z_{p^{2}}\right)$ is $K_{p-1}$.

Theorem 2.2: Let $R_{I}$ be a finite commutative ring such that $R_{l}=Z_{p} \times Z_{p^{2}}$ ( $p$ is a prime number). Let $G=\Gamma\left(R_{l}\right)$ be the zero-divisor graph with vertex set $Z\left(R_{l}\right) *$. Then number of vertices of $G=\Gamma\left(R_{l}\right)$ is $2 p^{2}-p-1, \Delta(G)=p^{2}-1$ and $\delta(G)=p-1$.

Proof: Let $R_{l}$ be a finite commutative ring such that $R_{l}=$ $Z_{p} \times Z_{p^{2}}\left(p\right.$ is a prime number). Let $R_{I}^{*}=R_{I}-\{0\}$. Then $R_{I} *$ can be partitioned into disjoint sets $A, B, C, D$ and $E$ such that $A=\left\{(u, 0): u \in Z_{p}^{*}\right\}, B=\left\{(0, v): v \in Z_{p^{2}}{ }^{*}\right.$ and $v \notin$ $\left.\mathrm{Z}\left(Z_{p^{2}}\right)^{*}\right\}, C=\left\{(0, w): w \in Z_{p^{2}}^{*}\right.$ and $\left.w \in \mathrm{Z}\left(Z_{p^{2}}\right)^{*}\right\}$, $D=\left\{(a, b): a \in Z_{p}{ }^{*}, b \in Z_{p^{2}}{ }^{*}\right.$ and $\left.b \in \mathrm{Z}\left(Z_{p^{2}}\right)^{*}\right\}$ and $E=$ $\left\{(c, d): c \in Z_{p}^{*}, d \in Z_{p^{2}}{ }^{*}\right.$ and $\left.d \notin \mathrm{Z}\left(Z_{p^{2}}\right)^{*}\right\}$. Clearly, all the elements in $A, B, C$ are non-zero zero-divisors. Let $(a, b) \in D$ and $(0, w) \in C$. Here $b, w \in \mathrm{Z}\left(Z_{p^{2}}\right)^{*}$. So, $p / b$ and $p / w$. This gives $p^{2} / b w$. Therefore, $(a, b)(0, w)=$ $(0,0)$. Hence, every element of $D$ is a non-zero zero-divisor. But product of any two elements of $E$ is not equal to zero. Also, product of any element of $E$ with any element of $A, B, C$ and $D$ is not equal to zero because, $c u \neq 0$ for $c, u \in Z_{p}{ }^{*}$, $d v \neq 0$ for $d, v \in Z_{p^{2}}{ }^{*}$ and $d, v \notin \mathrm{Z}\left(Z_{p^{2}}\right)^{*}, d w \neq 0$ for $d, w$ $\in Z_{p^{2}}{ }^{*}$ and $d \notin \mathrm{Z}\left(Z_{p^{2}}\right)^{*}, w \in \mathrm{Z}\left(Z_{p^{2}}\right)^{*}$ and $c a \neq 0$ for $c, a \in Z_{p}{ }^{*}$ respectively. So, no element of $E$ is a non-zero zero-divisor. Let $G=\Gamma\left(R_{l}\right)$ be the zero-divisor graph with vertex set $\mathrm{Z}\left(R_{l}\right)^{*}$. Then $\mathrm{Z}\left(R_{l}\right)^{*}$ can be partitioned into four disjoint sets $A, B, C$ and $D$. Now using the Lemma 2.1 we have $|A|=\left|Z_{p}^{*}\right|=p-1,|B|=\left|Z_{p^{2}}^{*}\right|-\left|Z\left(Z_{p^{2}}\right)^{*}\right|=$ $\left(p^{2}-1\right)-(p-1)=p^{2}-1-p+1=p^{2}-p,|C|=$ $\left|\mathrm{Z}\left(Z_{p^{2}}\right)^{*}\right|=p-1,|D|=\left|Z_{p} *\right|\left|\mathrm{Z}\left(Z_{p^{2}}\right)^{*}\right|=(p-1)$ $(p-1)=p^{2}-2 p+1$.

Therefore, $\left|\mathrm{Z}\left(R_{l}\right)^{*}\right|=|A|+|B|+|C|+|D|=(p-1)+$ $\left(p^{2}-p\right)+(p-1)+\left(p^{2}-2 p+1\right)=2 p^{2}-p-1$.

So, the number of vertices of $G=\Gamma\left(R_{l}\right)$ is $2 p^{2}-p-1$.

Let $s=(u, 0)$ be any vertex of $A$.

(i) Every vertex of $A$ is adjacent to every vertex of $B$. So, $s$ is adjacent to $p^{2}$ - $p$ vertices of $B$.

(ii) Every vertex of $A$ is adjacent to every vertex of $C$. So, $s$ is adjacent to $p-1$ vertices of $C$.

(iii) Any vertex of $A$ is not adjacent to any vertex of $D$ as $u a \neq 0$ for $u, a \in Z_{p}^{*}$.

Therefore, $\operatorname{deg}_{G}(s)=\left(p^{2}-p\right)+(p-1)=p^{2}-1$.

Let $t=(0, v)$ be any vertex of $B$.

(i) Every vertex of $B$ is adjacent to every vertex of $A$. So, $t$ is adjacent to $p-1$ vertices of $A$.

(ii) Any vertex of $B$ is not adjacent to any vertex of $C$ as $v w \neq 0$ for $v, w \in Z_{p^{2}}{ }^{*}$ and $v \notin \mathrm{Z}\left(Z_{p^{2}}\right)^{*}, w \in \mathrm{Z}\left(Z_{p^{2}}\right)^{*}$.

(iii) Any vertex of $B$ is not adjacent to any vertex of $D$ as $v b \neq 0$ for $v, b \in Z_{p^{2}}{ }^{*}$ and $v \notin \mathrm{Z}\left(Z_{p^{2}}\right)^{*}, b \in \mathrm{Z}\left(Z_{p^{2}}\right)^{*}$.

Therefore, $\operatorname{deg}_{G}(t)=p-1$.

Let $x=(0, w)$ be any vertex of $C$.

(i) Every vertex of $C$ is adjacent to every vertex of $A$. So, $x$ is adjacent to $p-1$ vertices of $A$.

(ii) Any two vertices of $C$ are adjacent to each other. So, $x$ is adjacent to $p-2$ vertices of $C$.

(iii) Every vertex of $C$ is adjacent to every vertex of $D$. So, $x$ is adjacent to $p^{2}-2 p+1$ vertices of $D$.

(iv) Any vertex of $C$ is not adjacent to any vertex of $B$ as $w v \neq 0$ for $w, v \in Z_{p^{2}}{ }^{*}$ and $w \in \mathrm{Z}\left(Z_{p^{2}}\right)^{*}, v \notin \mathrm{Z}\left(Z_{p^{2}}\right)^{*}$.

Therefore, $\operatorname{deg}_{G}(x)=(p-1)+(p-2)+\left(p^{2}-2 p+1\right)=p^{2}-2$.

Let $y=(a, b)$ be any vertex of $D$.

(i) Every vertex of $D$ is adjacent to every vertex of $C$. So, $y$ is adjacent to $p-1$ vertices of $C$.

(ii) Any vertex of $D$ is not adjacent to any vertex of $A$ as $a u \neq 0$ for $u, a \in Z_{p}^{*}$.

(iii) Any vertex of $D$ is not adjacent to any vertex of $B$ as $b v \neq 0$ for $b, v \in Z_{p^{2}}{ }^{*}$ and $b \in \mathrm{Z}\left(Z_{p^{2}}\right)^{*}, v \notin \mathrm{Z}\left(Z_{p^{2}}\right)^{*}$.

Therefore, $\operatorname{deg}_{G}(y)=p-1$.

Hence, we have $\Delta(G)=p^{2}-1$ and $\delta(G)=p-1$.

Theorem 2.3: Let $M_{l}$ be of the adjacency matrix for the zerodivisor graph $G=\Gamma\left(R_{l}\right)$ of $R_{l}=Z_{p} \times Z_{p^{2}}$ ( $p$ is a prime number). Then (i) determinant of $M_{1}$ is zero (ii) $M_{1}$ is symmetric and singular. 
Proof: Let $R_{l}$ be a finite commutative ring such that $R_{l}=$ $Z_{p} \times Z_{p^{2}}$ ( $p$ is a prime number). Let $G=\Gamma\left(R_{l}\right)$ be the zerodivisor graph with vertex set $V=\mathrm{Z}\left(R_{l}\right)^{*}$ and $M_{l}$ be the adjacency matrix for the zero-divisor graph of $R_{l}=$ $Z_{p} \times Z_{p^{2}}$.

(i) Since, at least two vertices of $G=\Gamma\left(R_{l}\right)$ are adjacent to same vertex of $G$, so $M_{l}$ contains at least two identical rows (eg. for $Z_{2} \times Z_{4}$ ). Therefore, the determinant of the adjacency matrix $M_{l}$ is zero.

(ii) Clearly $M_{l}$ is symmetric. Since, the determinant of the adjacency matrix $M_{l}$ is zero, $M_{l}$ is singular.

Theorem 2.4: Let $R_{l}$ be a finite commutative ring such that $R_{l}$ $=Z_{p} \times Z_{p^{2}}$ ( $p$ is a prime number). Let $G=\Gamma\left(R_{l}\right)$ be the zero-divisor graph with vertex set $V=Z\left(R_{I}\right)^{*}$. Then $n_{G}(V)=$ $2 \Delta(G)-\delta(G)$, where $n_{G}(V)$ is the neighborhood number, $\Delta(G)$ and $\delta(G)$ denote the maximum and minimum degree of $G$ respectively.

Proof: Let $R_{l}$ be a finite commutative ring such that $R_{l}=$ $Z_{p} \times Z_{p^{2}}\left(p\right.$ is a prime number). Let $G=\Gamma\left(R_{l}\right)$ be the zerodivisor graph with vertex set $V=\mathrm{Z}\left(R_{l}\right)^{*}$. Since, $G=\Gamma\left(R_{l}\right)$ is connected [1], we have $n_{G}(V)=\left|N_{G}(V)\right|=|V|=\left|Z\left(R_{1}\right)^{*}\right|$.

But from Theorem 2.2, we have $\left|Z\left(R_{1}\right)^{*}\right|=2 p^{2}-p-1$. Therefore, $n_{G}(V)=2 p^{2}-p-1$. This implies $n_{G}(V)=2\left(p^{2}-1\right)$ - $(p-1)$. Also, $\Delta(G)=p^{2}-1$ and $\delta(G)=p-1$ [from

Theorem 2.2]. This gives $n_{G}(V)=2 \Delta(G)-\delta(G)$.

\section{CONSTRUCTION OF ZERO -DIVISOR GRAPH FOR $\boldsymbol{R}_{2}=Z_{p} \times Z_{2 p} \quad(p$ IS AN ODD PRIME NUMBER):}

Secondly, we construct the zero-divisor graph for the ring $R_{2}=Z_{p} \times Z_{2 p}$ ( $p$ is an odd prime number) and analyze the graph. We start with the cases $p=3$ and $p=5$ and then generalize the cases.

Case 1: When $p=3$ we have $R_{2}=Z_{3} \times Z_{6}$

The ring $R_{2}$ has 13 non-zero zero-divisors. In this case $V=$ $\mathrm{Z}\left(R_{2}\right)^{*}=\{(1,0),(2,0),(1,2),(1,3),(1,4),(2,2),(2,3),(2,4),(0,1)$, $(0,2),(0,3),(0,4),(0,5)\}$ and the zero-divisor graph $G=\Gamma\left(R_{2}\right)$ is given by:

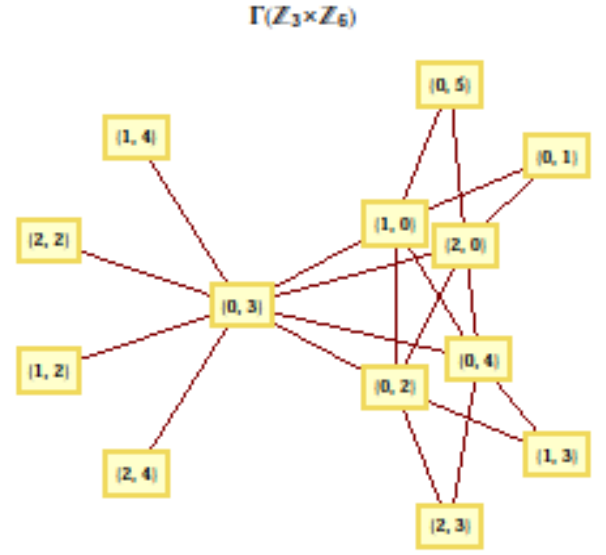

Fig: 3

The closed neighborhoods of the vertices are $N_{G}[(1,0)]=\{(0,1),(0,2),(0,3),(0,4),(0,5),(1,0)\}, N_{G}[(2,0)]=$ $\{(0,1),(0,2),(0,3),(0,4),(0,5),(2,0)\}, N_{G}[(1,2)]=\{(0,3),(1,2)\}$, $N_{G}[(1,3)]=\{(0,2),(0,4),(1,3)\}, N_{G}[(1,4)]=\{(0,3),(1,4)\}$, $N_{G}[(2,2)]=\{(0,3),(2,2)\}, N_{G}[(2,3)]=\{(0,2),(0,4),(2,3)\}$, $N_{G}[(2,4)]=\{(0,3),(2,4)\}, N_{G}[(0,1)]=\{(1,0),(2,0),(0,1)\}$, $N_{G}[(0,2)]=\{(1,0),(2,0),(1,3),(2,3),(0,3),(0,2)\}, N_{G}[(0,3)]=$ $\{(1,0),(2,0),(1,2),(1,4),(2,2),(2,4),(0,2),(0,4),(0,3)\}, N_{G}[(0,4)]$ $=\{(1,0),(2,0),(1,3),(2,3),(0,3),(0,4)\}, N_{G}[(0,5)]=\{(1,0),(2,0)$, $(0,5)\}$. The neighborhood of $V$ is given by $N_{G}(V)=\{(1,0)$, $(2,0),(1,2),(1,3),(1,4),(2,2),(2,3),(2,4),(0,1),(0,2),(0,3)$, $(0,4),(0,5)\}$. The maximum degree is $\Delta(G)=8$ and minimum degree is $\delta(G)=1$. The adjacency matrix for the zerodivisor graph of $R_{2}=Z_{3} \times Z_{6}$ is $M_{2}=\left[\begin{array}{cc}O_{8 \times 8} & A_{8 \times 5} \\ A^{T}{ }_{5 \times 8} & B_{5 \times 5}\end{array}\right]_{13 \times 13}$, where $A_{8 \times 5}=\left[\begin{array}{ccccc}1 & 1 & 1 & 1 & 1 \\ 1 & 1 & 1 & 1 & 1 \\ 0 & 0 & 1 & 0 & 0 \\ 0 & 1 & 0 & 1 & 0 \\ 0 & 0 & 1 & 0 & 0 \\ 0 & 0 & 1 & 0 & 0 \\ 0 & 1 & 0 & 1 & 0 \\ 0 & 0 & 1 & 0 & 0\end{array}\right], B_{5 \times 5}=\left[\begin{array}{ccccc}0 & 0 & 0 & 0 & 0 \\ 0 & 0 & 1 & 0 & 0 \\ 0 & 1 & 0 & 1 & 0 \\ 0 & 0 & 1 & 0 & 0 \\ 0 & 0 & 0 & 0 & 0\end{array}\right]$, $O_{8 \times 8}$ is the zero matrix and $A^{T}{ }_{5 \times 8}$ is the transpose of $A_{8 \times 5}$.

\section{Properties of adjacency matrix $M_{2}$ :}

(i) The determinant of the adjacency matrix $M_{2}$ corresponding to $G=\Gamma\left(R_{2}\right)$ is 0 .

(ii) The rank of the adjacency matrix $M_{2}$ corresponding to $G$ $=\Gamma\left(R_{2}\right)$ is 2 .

(iii) The adjacency matrix $M_{2}$ corresponding to $G=\Gamma\left(R_{2}\right)$ is symmetric and singular.

Case 2: When $p=5$ we have $R_{2}=Z_{5} \times Z_{10}$

The ring $R_{2}$ has 33 non-zero zero-divisors. In this case $V=$ $\mathrm{Z}\left(R_{2}\right)^{*}=\{(1,0),(2,0),(3,0),(4,0),(1,2),(1,4),(1,5),(1,6),(1,8)$, $(2,2),(2,4),(2,5),(2,6),(2,8),(3,2),(3,4),(3,5),(3,6),(3,8),(4,2)$, $(4,4),(4,5),(4,6),(4,8),(0,1),(0,2),(0,3),(0,4),(0,5),(0,6)$, $(0,7),(0,8),(0,9)\}$ and the zero-divisor graph $G=\Gamma\left(R_{2}\right)$ is given by: 


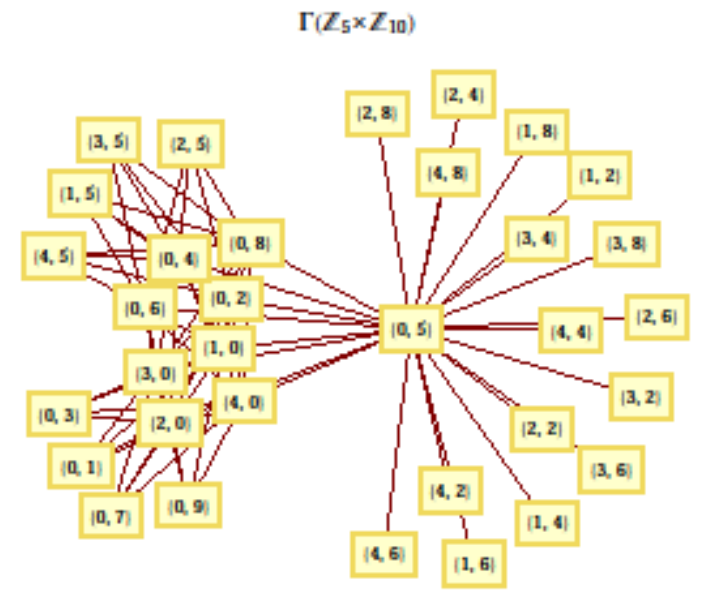

Fig: 4

The closed neighborhoods of the vertices are $N_{G}[(1,0)]=\{(0,1),(0,2),(0,3),(0,4),(0,5),(0,6),(0,7),(0,8)$, $(0,9),(1,0)\}, N_{G}[(2,0)]=\{(0,1),(0,2),(0,3),(0,4),(0,5),(0,6)$, $(0,7),(0,8),(0,9),(2,0)\}, N_{G}[(3,0)]=\{(0,1),(0,2),(0,3),(0,4)$, $(0,5),(0,6),(0,7),(0,8),(0,9),(3,0)\}, N_{G}[(4,0)]=\{(0,1),(0,2)$, $(0,3),(0,4),(0,5),(0,6),(0,7),(0,8),(0,9),(4,0)\}, \quad N_{G}[(1,2)]=$ $\{(0,5),(1,2)\}, \quad N_{G}[(1,4)]=\{(0,5),(1,4)\}, \quad N_{G}[(1,5)]=\{(0,2)$, $(0,4),(0,6),(0,8),(1,5)\}, N_{G}[(1,6)]=\{(0,5),(1,6)\}, N_{G}[(1,8)]$ $=\{(0,5),(1,8)\}, \quad N_{G}[(2,2)]=\{(0,5),(2,2)\}, N_{G}[(2,4)]=\{(0,5)$, $(2,4)\}\}, N_{G}[(2,5)]=\{(0,2),(0,4),(0,6),(0,8),(2,5)\}, N_{G}[(2,6)]$ $=\{(0,5),(2,6)\}, N_{G}[(2,8)]=\{(0,5),(2,8)\}, N_{G}[(3,2)]=\{(0,5)$, $(3,2)\}, \quad N_{G}[(3,4)]=\{(0,5),(3,4)\}, \quad N_{G}[(3,5)]=\{(0,2),(0,4)$, $(0,6),(0,8),(3,5)\}, N_{G}[(3,6)]=\{(0,5),(3,6)\}, N_{G}[(3,8)]=\{(0,5)$, $\left.(3,8)\}, \quad N_{G}[(4,2)]=\{(0,5),(4,2)\}\right\}, \quad N_{G}[(4,4)]=\{(0,5),(4,4)\}$, $N_{G}[(4,5)]=\{(0,2),(0,4),(0,6),(0,8),(4,5)\}, N_{G}[(4,6)]=\{(0,5)$, $(4,6)\}, N_{G}[(4,8)]=\{(0,5),(4,8)\}, \quad N_{G}[(0,1)]=\{(1,0),(2,0)$, $(3,0),(4,0),(0,1)\}, N_{G}[(0,2)]=\{(1,0),(2,0),(3,0),(4,0),(0,5)$, $(1,5),(2,5),(3,5),(4,5),(0,2)\}, N_{G}[(0,3)]=\{(1,0),(2,0),(3,0)$, $(4,0),(0,3)\}, \quad N_{G}[(0,4)]=\{(1,0),(2,0),(3,0),(4,0),(0,5),(1,5)$, $(2,5),(3,5),(4,5),(0,4)\}, N_{G}[(0,5)]=\{(1,0),(2,0),(3,0),(4,0)$, $(1,2),(1,4),(1,6),(1,8),(2,2),(2,4),(2,6),(2,8),(3,2),(3,4)$, $(3,6),(3,8),(4,2),(4,4),(4,6),(4,8),(0,2),(0,4),(0,6),(0,8)$, $(0,5)\}, \quad N_{G}[(0,6)]=\{(1,0),(2,0),(3,0),(4,0),(1,5),(2,5),(3,5)$, $(4,5),(0,5),(0,6)\}, N_{G}[(0,7)]=\{(1,0),(2,0),(3,0),(4,0),(0,7)\}$, $N_{G}[(0,8)]=\{(1,0),(2,0),(3,0),(4,0),(1,5),(2,5),(3,5),(4,5)$, $(0,5),(0,8)\}, N_{G}[(0,9)]=\{(1,0),(2,0),(3,0),(4,0),(0,9)\}$.The neighborhood of $V$ is given by $N_{G}(V)=\{(1,0),(2,0),(3,0)$, $(4,0),(1,2),(1,4),(1,5),(1,6),(1,8),(2,2),(2,4),(2,5),(2,6)$, $(2,8),(3,2),(3,4),(3,5),(3,6),(3,8),(4,2),(4,4),(4,5),(4,6),(4,8)$, $(0,1),(0,2),(0,3),(0,4),(0,5),(0,6),(0,7),(0,8),(0,9)\}$. The maximum degree is $\Delta(G)=24$ and minimum degree is $\delta(G)=1$. The adjacency matrix for the zero-divisor graph of $R_{2}=Z_{5} \times Z_{10}$ is $M_{2}=\left[\begin{array}{cc}O_{24 \times 24} & A_{24 \times 9} \\ A^{T} & B_{9 \times 24}\end{array}\right]_{33 \times 33}$,

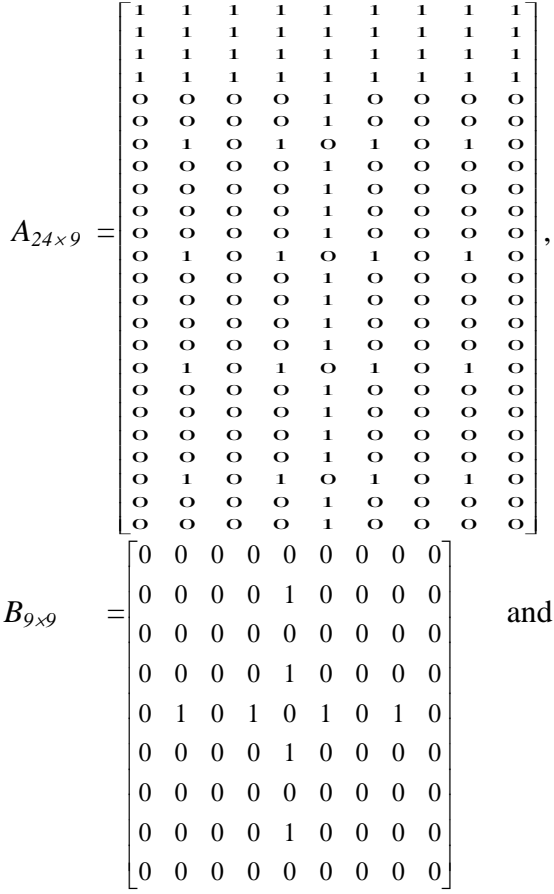

$O_{24 \times 24}$ is the zero matrix and $A^{T}{ }_{9 \times 24}$ is the transpose of $A_{24 \times 9}$.

\section{Properties of adjacency matrix $M_{2}$ :}

(i) The determinant of the adjacency matrix $M_{2}$ corresponding to $G=\Gamma\left(R_{2}\right)$ is 0 .

(ii) The rank of the adjacency matrix $M_{2}$ corresponding to $G=\Gamma\left(R_{2}\right)$ is 2 .

(iii) The adjacency matrix $M_{2}$ corresponding to $G=\Gamma\left(R_{2}\right)$ is symmetric and singular.

\section{Generalization for $R_{2}=Z_{p} \times Z_{2 p}(p$ is an odd prime number):}

Lemma 3.1: The number of vertices of $G=\Gamma\left(Z_{2 p}\right)$ is $p$ and $G=\Gamma\left(Z_{2 p}\right)$ is $K_{1, p-1}$, where $p$ is an odd prime number.

Proof: The multiples of 2 less than $2 p$ are $2,4,6, \ldots \ldots$, $2(p-1)$. The non-zero zero-divisors of $Z_{2 p}$ are $p$ and 2, 4, $6, \ldots \ldots, 2(p-1)$. If $G=\Gamma\left(Z_{2 p}\right)$ is the zero-divisor graph of $Z_{2 p}$, then the vertices of $G=\Gamma\left(Z_{2 p}\right)$ are the non-zero zero-divisors of $Z_{2 p}$. So, the vertex set of $G=\Gamma\left(Z_{2 p}\right)$ is $\mathrm{Z}\left(Z_{2 p}\right)^{*}$ and $p$ and $2,4,6, \ldots \ldots, 2(p-1)$ are the vertices of $\Gamma\left(Z_{2 p}\right)$. Hence, the number of vertices of $G=\Gamma\left(Z_{2 p}\right)$ is $p$. Also, in $G=\Gamma\left(Z_{2 p}\right), p$ is adjacent to remaining vertices 2, 4, $6, \ldots \ldots, 2(p-1)$. This gives $G=\Gamma\left(Z_{2 p}\right)$ is $K_{1, p-1}$.

Theorem 3.2: Let $R_{2}$ be a finite commutative ring such that $R_{2}=$ $Z_{p} \times Z_{2 p}$ ( $p$ is an odd prime number). Let $G=\Gamma\left(R_{2}\right)$ be the zero-divisor graph with vertex set $Z\left(R_{2}\right) *$. Then number of vertices of $G=\Gamma\left(R_{2}\right)$ is $p^{2}+2 p-2, \Delta(G)=p^{2}-1$ and $\delta(G)=1$. 
Proof: Let $R_{2}$ be a finite commutative ring such that that $R_{2}=$ $Z_{p} \times Z_{2 p}$ ( $p$ is an odd prime number). Let $R_{2}^{*}=R_{2}-\{0\}$. Then $R_{2} *$ can be partitioned into disjoint sets $A, B, C, D$ and $E$ such that $A=\left\{(u, 0): u \in Z_{p}{ }^{*}\right\}, B=\left\{(0, v): v \in Z_{2 p}{ }^{*}\right.$ and $\left.v \notin \mathrm{Z}\left(Z_{2 p}\right)^{*}\right\}, C=\left\{(0, w): w \in Z_{2 p}{ }^{*}\right.$ and $\left.w \in \mathrm{Z}\left(Z_{2 p}\right)^{*}\right\}$, $D=\left\{(a, b): a \in Z_{p}{ }^{*}, b \in Z_{2 p}{ }^{*}\right.$ and $\left.b \in \mathrm{Z}\left(Z_{2 p}\right)^{*}\right\}$ and $E=$ $\left\{(c, d): c \in Z_{p}{ }^{*}, d \in Z_{2 p}{ }^{*}\right.$ and $\left.d \notin \mathrm{Z}\left(Z_{2 p}\right)^{*}\right\}$ respectively. Clearly, all the elements in $A, B, C$ are non-zero zero-divisors. Let $(a, b) \in D$. Then $(a, b)$ is of the form either $(a, p)$ or $(a, q)$, where $q=2 m, 1 \leq m \leq p-1$. Again let $(0, w) \in C$. Similarly, $(0, w)$ is of the form either $(0, p)$ or $(0, q)$, where $q=2 m, 1 \leq m \leq$ $p-1$. Now $p / p$ and $2 / q$. This gives $2 p / p q$. Therefore, $(a, p)(0, q)=(0,0)$ and $(a, q)(0, p)=(0,0)$. Hence, every element of $D$ is a non-zero zero-divisor. But product of any two elements of $E$ is not equal to zero. Also, product of any element of $E$ with any element of $A, B, C$ and $D$ is not equal to zero because, $c u \neq 0$ for $c, u \in Z_{p}{ }^{*}, d v \neq 0$ for $d, v \in Z_{2 p}{ }^{*}$ and $d$, $v \notin \mathrm{Z}\left(Z_{2 p}\right)^{*}, d w \neq 0$ for $d, w \in Z_{2 p}{ }^{*}$ and $d \notin \mathrm{Z}\left(Z_{2 p}\right)^{*}$, $w \in \mathrm{Z}\left(Z_{2 p}\right)^{*}$ and $\quad c a \neq 0$ for $c, a \in Z_{p}^{*}$ respectively. So, no element of $E$ is a non-zero zero-divisor. Let $G=\Gamma\left(R_{2}\right)$ be the zero-divisor graph with vertex set $\mathrm{Z}\left(R_{2}\right)^{*}$. Then $\mathrm{Z}\left(R_{2}\right)^{*}$ can be partitioned into four disjoint sets $A, B, C$ and $D$. Now using the

Lemma 3.1 we have $|A|=\left|Z_{p}{ }^{*}\right|=p-1, \quad|B|=\left|Z_{2 p}{ }^{*}\right|-$

$\left|\mathrm{Z}\left(Z_{2 p}\right)^{*}\right|=(2 p-1)-p=p-1,|C|=\left|\mathrm{Z}\left(Z_{2 p}\right)^{*}\right|=p$,

$|D|=\left|Z_{p} *\right|\left|\mathrm{Z}\left(Z_{2 p}\right)^{*}\right|=(p-1) p=p^{2}-p$.

Therefore, $\left|\mathrm{Z}\left(R_{2}\right) *\right|=|A|+|B|+|C|+|D|=(p-1)+$ $(p-1)+p+\left(p^{2}-p\right)=p^{2}+2 p-2$

So, the number of vertices of $G=\Gamma\left(R_{2}\right)$ is $p^{2}+2 p-2$.

Let $s=(u, 0)$ be any vertex of $A$.

(i) Every vertex of $A$ is adjacent to every vertex of $B$. So, $s$ is adjacent to $p-1$ vertices of $B$.

(ii) Every vertex of $A$ is adjacent to every vertex of $C$. So, $s$ is adjacent to $p$ vertices of $C$.

(iii) Any vertex of $A$ is not adjacent to any vertex of $D$ as $u a \neq 0$ for $u, a \in Z_{p}^{*}$.

Therefore, $\operatorname{deg}_{G}(s)=(p-1)+p=2 p-1$.

Let $t=(0, v)$ be any vertex of $B$.

(i) Every vertex of $B$ is adjacent to every vertex of $A$. So, $t$ is adjacent to $p-1$ vertices of $A$

(ii) Any vertex of $B$ is not adjacent to any vertex of $C$ as $v w \neq 0$ for and $v, w \in Z_{2 p}{ }^{*}$ and $v \notin \mathrm{Z}\left(Z_{2 p}\right)^{*}, w \in \mathrm{Z}\left(Z_{2 p}\right)^{*}$

(iii) Any vertex of $B$ is not adjacent to any vertex of $D$ as $v b \neq 0$ for $v, b \in Z_{2 p}{ }^{*}$ and $v \notin \mathrm{Z}\left(Z_{2 p}\right)^{*}, b \in \mathrm{Z}\left(Z_{2 p}\right)^{*}$.

Therefore, $\operatorname{deg}_{G}(t)=p-1$

Let $x=(0, w)$ be any vertex of $C$. Then either $x=(0, p)$ or $x=(0, q)$, where $q=2 m, 1 \leq m \leq p-1$

(i) Every vertex of $C$ is adjacent to every vertex of $A$. So, $x$ is adjacent to $p-1$ vertices of $A$.

(ii) Case 1: If $x=(0, p)$, then it is adjacent to $p-1$ vertices of $C$.
Case 2: If $x=(0, q)$, then it is adjacent to only one vertex of

(iii) Case 1: If $x=(0, p)$, then it is adjacent to $\left|Z_{p} *\right|^{2}=(p-1)^{2}$ vertices of $D$.

Case 2: If $x=(0, q)$, then it is adjacent to $\left|Z_{p} *\right|=p-1$ vertices of $D$.

(iv) Any vertex of $C$ is not adjacent to any vertex of $B$ as $w v \neq 0$ for $w, v \in Z_{2 p}{ }^{*}$ and $w \notin \mathrm{Z}\left(Z_{2 p}\right)^{*}, v \in \mathrm{Z}\left(Z_{2 p}\right)^{*}$.

Therefore, if $x=(0, p)$, then $\operatorname{deg}_{G}(x)=(p-1)+(p-1)+(p-1)^{2}$ $=p^{2}-1$ and if $x=(0, q)$, then $\operatorname{deg}_{G}(x)=(p-1)+1+(p-1)=$ $2 p-1$.

Let $y=(a, b)$ be any vertex of $D$. Then either $y=(a, p)$ or $y=$ $(a, q)$, where $a \in Z_{p}^{*}$ and $q=2 m, 1 \leq m \leq p-1$

(i) Case 1: If $y=(a, p)$, then it is adjacent to $p-1$ vertices of $C$. C.

Case 2: If $y=(a, q)$, then it is adjacent to only one vertex of

(ii) Any vertex of $D$ is not adjacent to any vertex of $A$ as $a u \neq 0$ for $u, a \in Z_{p}{ }^{*}$

(iii) Any vertex of $D$ is not adjacent to any vertex of $B$ as $b v \neq 0$ for $b, v \in Z_{2 p}{ }^{*}$ and $b \in \mathrm{Z}\left(Z_{2 p}\right)^{*}, v \notin \mathrm{Z}\left(Z_{2 p}\right)^{*}$.

Therefore, if $y=(a, p)$, then $\operatorname{deg}_{G}(y)=p-1$ and if $y=(a, q)$, then $\operatorname{deg}_{G}(y)=1$.

Hence, we have $\Delta(G)=p^{2}-1$ and $\delta(G)=1$.

Theorem 3.3: Let $M_{2}$ be of the adjacency matrix for the zerodivisor graph $G=\Gamma\left(R_{2}\right)$ of $R_{2}=Z_{p} \times Z_{2 p}$ ( $p$ is an odd prime number). Then (i) determinant of $M_{2}$ is zero (ii) $M_{2}$ is symmetric and singular.

\section{Proof: Follows from Theorem 2.3.}

Theorem 3.4: Let $R_{2}$ be a finite commutative ring such that $R_{2}=Z_{p} \times Z_{2 p}$ ( $p$ is an odd prime number). Let $G=\Gamma\left(R_{2}\right)$ be the zero-divisor graph with vertex set $V=\mathrm{Z}\left(R_{2}\right)^{*}$.Then $n_{G}(V)=2 p+\Delta(G)-\delta(G)$, where $n_{G}(V)$ is the neighborhood number, $\Delta(G)$ and $\delta(G)$ denote the maximum and minimum degree of $G$ respectively.

.Proof: Let $R_{2}$ be a finite commutative ring such that $R_{2}=$ $Z_{p} \times Z_{2 p}$ ( $p$ is an odd prime number). Let $G=\Gamma\left(R_{2}\right)$ be the zero-divisor graph with vertex set $V=\mathrm{Z}\left(R_{2}\right) *$. Since, $G=\Gamma\left(R_{2}\right)$ is connected [1], we have $n_{G}(V)=\left|N_{G}(V)\right|=|V|=\left|Z\left(R_{2}\right)^{*}\right|$. But from Theorem 3.2, we have $\left|Z\left(R_{2}\right)^{*}\right|=p^{2}+2 p-2$. Therefore, $n_{G}(V)=p^{2}+2 p-2$.This implies $n_{G}(V)=2 p+\left(p^{2}-1\right)$ -1. Also $\Delta(G)=p^{2}-1$ and $\delta(G)=1$ [from Theorem 3.2]. This gives $n_{G}(V)=2 p+\Delta(G)-\delta(G)$.

Remark: If $p=2$, then $R_{2}=Z_{2} \times Z_{4}$. So, this case coincides with the case 1 of section 2. 


\section{CONSTRUCTION OF ZERO -DIVISOR GRAPH FOR $\boldsymbol{R}_{3}=Z_{p} \times Z_{p^{2}-2}$ ( FOR THAT ODD PRIME $p$ FOR WHICH $p^{2}-2$ IS A PRIME NUMBER):}

Thirdly, we construct the zero-divisor graph for the ring $R_{3}=Z_{p} \times Z_{p^{2}-2}$ (for that odd prime $p$ for which $p^{2}-2$ is a prime number) and analyze the graph. We start with the cases $p=3$ and $p=5$ and then generalize the cases.

Case 1: When $p=3$ we have $R_{3}=Z_{3} \times Z_{7}$

The ring $R_{3}$ has 8 non-zero zero-divisors. In this case $V=$ $\mathrm{Z}\left(R_{3}\right)^{*}=\{(1,0),(2,0),(0,1),(0,2),(0,3),(0,4),(0,5),(0,6)\}$ and the zero-divisor graph $G=\Gamma\left(R_{3}\right)$ is given by:

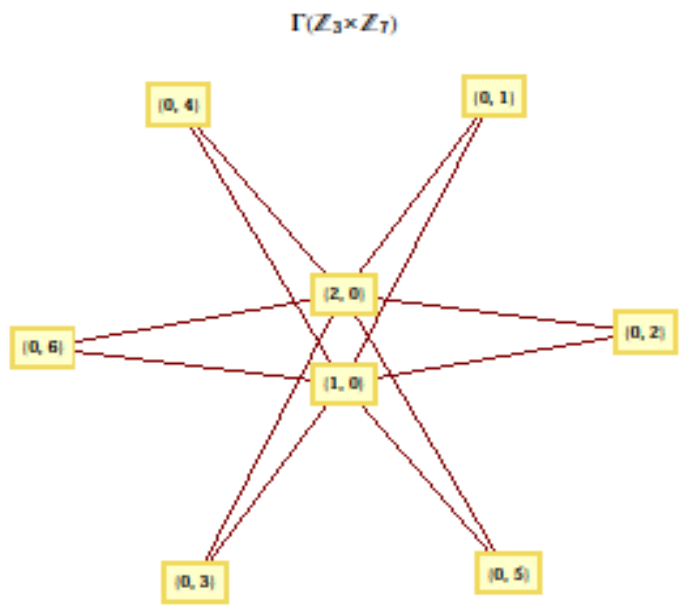

Fig: 5

The closed neighborhoods of the vertices are $N_{G}[(1,0)]=\{(0,1),(0,2),(0,3),(0,5),(0,6),(1,0)\}, N_{G}[(2,0)]=$ $\{(0,1),(0,2),(0,3),(0,5),(0,6),(2,0)\}, N_{G}[(0,1)]=\{(1,0),(2,0)$, $(0,1)\}, N_{G}[(0,2)]=\{(1,0),(2,0),(0,2)\}, N_{G}[(0,3)]=\{(1,0)$, $(2,0),(0,3)\}, N_{G}[(0,4)]=\{(1,0),(2,0),(0,4)\}, N_{G}[(0,5)]=\{(1,0)$, $(2,0),(0,5)\}, N_{G}[(0,6)]=\{(1,0),(2,0),(0,6)\}$. The neighborhood of $V$ is given by $N_{G}(V)=\{(1,0),(2,0),(0,1),(0,2),(0,3),(0,4)$, $(0,5),(0,6)\}$. The maximum degree is $\Delta(G)=6$ and minimum degree is $\delta(G)=2$. The adjacency matrix for the zerodivisor graph of $R_{3}=Z_{3} \times Z_{7}$ is $M_{3}=\left[\begin{array}{cc}O_{2 \times 2} & A_{2 \times 6} \\ A^{T}{ }_{6 \times 2} & O_{6 \times 6}\end{array}\right]_{8 \times 8}$ where all the entries of $A_{2 \times 6}$ is $1, A^{T}{ }_{6 \times 2}$ is the transpose of $A_{2 \times 6}$ and $O_{2 \times 2}, O_{6 \times 6}$ are the zero matrices.

\section{Properties of adjacency matrix $M_{3}$ :}

(i) The determinant of the adjacency matrix $M_{3}$ corresponding to $G=\Gamma\left(R_{3}\right)$ is 0 .

(ii) The rank of the adjacency matrix $M_{3}$ corresponding to $G$ $=\Gamma\left(R_{3}\right)$ is 2 . (iii) The adjacency matrix $M_{3}$ corresponding to $G=\Gamma\left(R_{3}\right)$ is symmetric and singular.

Case 2: When $p=5$ we have $R_{3}=Z_{5} \times Z_{23}$.

The ring $R_{3}$ has 26 non-zero zero-divisors. In this case $V=$ $\mathrm{Z}\left(R_{3}\right)^{*}=\{(1,0),(2,0),(3,0),(4,0),(0,1),(0,2),(0,3),(0,4),(0,5)$, $(0,6),(0,7),(0,8),(0,9),(0,10),(0,11),(0,12),(0,13),(0,14)$, $(0,15),(0,16),(0,17),(0,18),(0,19),(0,20),(0,21),(0,22)\}$ and the zero-divisor graph $G=\Gamma\left(R_{3}\right)$ is given by:

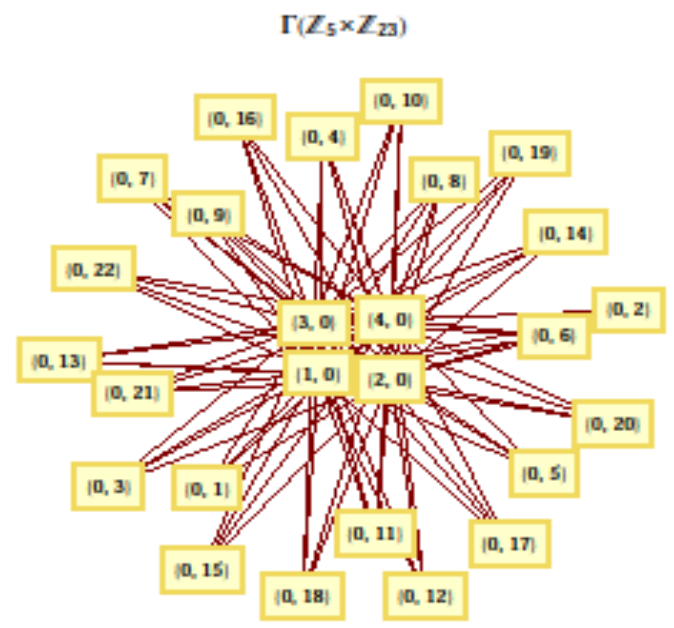

Fig: 6

The closed neighborhoods of the vertices are $N_{G}[(1,0)]=\{(0,1),(0,2),(0,3),(0,4),(0,5),(0,6),(0,7),(0,8)$, $(0,9),(0,10),(0,11),(0,12),(0,13),(0,14),(0,15),(0,16)$ $(0,17),(0,18)\},(0,19),(0,20),(0,21),(0,22),(1,0)\}, N_{G}[(2,0)]=$ $\{(0,1),(0,2),(0,3),(0,4),(0,5),(0,6),(0,7),(0,8),(0,9),(0,10)$, $(0,11),(0,12),(0,13),(0,14),(0,15),(0,16),(0,17),(0,18)\}$, $(0,19),(0,20),(0,21),(0,22),(2,0)\}, N_{G}[(3,0)]=\{(0,1),(0,2)$, $(0,3),(0,4),(0,5),(0,6),(0,7),(0,8),(0,9),(0,10),(0,11),(0,12)$, $(0,13),(0,14),(0,15),(0,16),(0,17),(0,18)\},(0,19),(0,20)$, $(0,21),(0,22),(3,0)\}, N_{G}[(4,0)]=\{(0,1),(0,2),(0,3),(0,4)$, $(0,5),(0,6),(0,7),(0,8),(0,9),(0,10),(0,11),(0,12),(0,13)$, $(0,14),(0,15),(0,16),(0,17),(0,18)\},(0,19),(0,20),(0,21)$, $(0,22),(4,0)\}, N_{G}[(0,1)]=\{(1,0),(2,0),(3,0),(4,0),(0,1)\}$, $N_{G}[(0,2)]=\{(1,0),(2,0),(3,0),(4,0),(0,2)\}, \quad N_{G}[(0,3)]=$ $\{(1,0),(2,0),(3,0),(4,0),(0,3)\}, \quad N_{G}[(0,4)]=\{(1,0),(2,0)$, $(3,0),(4,0),(0,4)\}, N_{G}[(0,5)]=\{(1,0),(2,0),(3,0),(4,0),(0,5)\}$, $N_{G}[(0,6)]=\{(1,0),(2,0),(3,0),(4,0),(0,6)\}, N_{G}[(0,7)]=\{(1,0)$, $(2,0),(3,0),(4,0),(0,7)\}, N_{G}[(0,8)]=\{(1,0),(2,0),(3,0),(4,0)$, $(0,8)\}, N_{G}[(0,9)]=\{(1,0),(2,0),(3,0),(4,0),(0,9)\}, N_{G}[(0,10)]$ $=\{(1,0),(2,0),(3,0),(4,0),(0,10)\}, N_{G}[(0,11)]=\{(1,0),(2,0)$, $(3,0),(4,0),(0,11)\}, \quad N_{G}[(0,12)]=\{(1,0),(2,0),(3,0),(4,0)$, $(0,12)\}, \quad N_{G}[(0,13)]=\{(1,0), \quad(2,0), \quad(3,0),(4,0),(0,13)\}$, $N_{G}[(0,14)]=\{(1,0),(2,0),(3,0),(4,0),(0,14)\}, N_{G}[(0,15)]=$ $\{(1,0),(2,0),(3,0),(4,0),(0,15)\}, N_{G}[(0,16)]=\{(1,0),(2,0)$, $(3,0),(4,0),(0,16)\}, N_{G}[(0,17)]=\{(1,0),(2,0),(3,0),(4,0)$, $(0,17)\}, \quad N_{G}[(0,18)]=\{(1,0),(2,0),(3,0),(4,0),(0,18)\}$, $N_{G}[(0,19)]=\{(1,0),(2,0),(3,0),(4,0),(0,19)\}, \quad N_{G}[(0,20)]=$ $\{(1,0),(2,0),(3,0),(4,0),(0,20)\}, N_{G}[(0,21)]=\{(1,0),(2,0)$, $(3,0),(4,0),(0,21)\}, N_{G}[(0,22)]=\{(1,0),(2,0),(3,0),(4,0)$, $(0,22)\}$. The neighborhood of $V$ is given by $N_{G}(V)=\{(1,0)$, $(2,0),(3,0),(4,0),(0,1),(0,2),(0,3),(0,4),(0,5),(0,6),(0,7)$, $(0,8),(0,9),(0,10),(0,11),(0,12),(0,13),(0,14),(0,15),(0,16)$, $(0,17),(0,18),(0,19),(0,20),(0,21),(0,22)\}$. The maximum 
degree is $\Delta(G)=22$ and minimum degree is $\delta(G)=4$. The adjacency matrix for the zero-divisor graph of $R_{3}=Z_{5} \times Z_{23}$ is $M_{3}=\left[\begin{array}{cc}O_{4 \times 4} & A_{4 \times 22} \\ A^{T}{ }_{22 \times 4} & O_{22 \times 22}\end{array}\right]_{26 \times 26}$ where all the entries of $A_{4 \times 22}$ is $1, \quad A_{22 \times 4}^{T}$ is the transpose of $A_{4 \times 22}$ and $O_{4 \times 4}, O_{22 \times 22}$ are the zero matrices.

\section{Properties of adjacency matrix $M_{3}$ :}

(i) The determinant of the adjacency matrix $M_{3}$ corresponding to $G=\Gamma\left(R_{3}\right)$ is 0 .

(ii) The rank of the adjacency matrix $M_{3}$ corresponding to $G$ $=\Gamma\left(R_{3}\right)$ is 2 .

(iii) The adjacency matrix $M_{3}$ corresponding to $G=\Gamma\left(R_{3}\right)$ is symmetric and singular.

\section{Generalization for $\boldsymbol{R}_{3}=Z_{p} \times Z_{p^{2}-2}$ (for that odd prime $p$ for which $p^{2}-2$ is a prime number):}

Theorem 4.1: Let $R_{3}$ be a finite commutative ring such that $R_{3}=$ $Z_{p} \times Z_{p^{2}-2}$ (for that odd prime $p$ for which $p^{2}-2$ is a prime number). Let $G=\Gamma\left(R_{3}\right)$ be the zero-divisor graph with vertex set $\mathrm{Z}\left(R_{3}\right)^{*}$. Then number of vertices of $G=\Gamma\left(R_{3}\right)$ is $p^{2}+p-4$, $\Delta(G)=p^{2}-3$ and $\delta(G)=p-1$.

Proof: Let $R_{3}$ be a finite commutative ring such that that $R_{3}=$ $Z_{p} \times Z_{p^{2}-2}$ (for that odd prime $p$ for which $p^{2}-2$ is a prime number). Let $R_{3}^{*}=R_{3}-\{0\}$. Then $R_{3}^{*}$ can be partitioned into disjoint sets $A, B$ and $C$ such that $A=\left\{(u, 0): u \in Z_{p}^{*}\right\}, B=$ $\left\{(0, v): v \in Z_{p^{2}-2}^{*}\right\}$ and $C=\left\{(a, b): a \in Z_{p}^{*}\right.$ and $b \in Z_{p^{2}-2}^{*}$ respectively. Clearly, all the elements of $A$ and $B$ are non-zero zero-divisors. But product of any two elements of $C$ is not equal to zero. Also, product of any element of $C$ with any element of $A$ and $B$ is not equal to zero because, $a u \neq 0$ for $a, u \in Z_{p}^{*}, \quad b v \neq 0$ for $b, v \in Z_{p^{2}-2}^{*}$ respectively. So, no element of $C$ is a non-zero zero-divisor. Let $G=\Gamma\left(R_{3}\right)$ be the zero-divisor graph with vertex set $\mathrm{Z}\left(R_{3}\right)^{*}$. Then $\mathrm{Z}\left(R_{3}\right)^{*}$ can be partitioned into two disjoint sets $A$ and $B$. Now, $|A|=\left|Z_{p}^{*}\right|=$ $p-1$ and $|B|=\left|Z_{p^{2}-2}^{*}\right|=p^{2}-3$. Therefore, $\left|Z\left(R_{3}\right)^{*}\right|=$ $|A|+|B|=\left|Z_{p}^{*}\right|+\left|Z_{p^{2}-2}^{*}\right|=(p-1)+\left(p^{2}-3\right)=p^{2}+p-4$. So, the number of vertices of $G=\Gamma\left(R_{3}\right)$ is $p^{2}+p-4$.

Let $x=(u, 0)$ be any vertex of $A$.

(i) Every vertex of $A$ is adjacent to every vertex of $B$. So, $x$ is adjacent to $p^{2}-3$ vertices of $B$. Therefore, $\operatorname{deg}_{G}(x)=p^{2}-3$.

Let $y=(0, v)$ be any vertex of $B$.

(i) Every vertex of $B$ is adjacent to every vertex of $A$. So, $y$ is adjacent to $p-1$ vertices of $A$. Therefore, $\operatorname{deg}_{G}(y)=p-1$.

Hence, we have $\Delta(G)=p^{2}-3$ and $\delta(G)=p-1$.
Theorem 4 .2: Let $M_{3}$ be of the adjacency matrix for the zerodivisor graph $G=\Gamma\left(R_{3}\right)$ of $R_{3}=Z_{p} \times Z_{p^{2}-2}$ (for that odd prime $p$ for which $p^{2}-2$ is a prime number). Then (i) determinant of $M_{3}$ is zero (ii) $M_{3}$ is symmetric and singular.

Proof: Follows from Theorem 2.3.

Theorem 4.3: Let $R_{3}$ be a finite commutative ring such that $R_{3}$ $=Z_{p} \times Z_{p^{2}-2}$ (for that odd prime $p$ for which $p^{2}-2$ is a prime number). Let $G=\Gamma\left(R_{3}\right)$ be the zero-divisor graph with vertex set $V=\mathrm{Z}\left(R_{3}\right)^{*}$. Then $n_{G}(V)=\Delta(G)+\delta(G)$, where $n_{G}(V)$ is the neighborhood number, $\Delta(G)$ and $\delta(G)$ denote the maximum and minimum degree of $G$ respectively.

Proof: Let $R_{3}$ be a finite commutative ring such that $R_{3}$ $=Z_{p} \times Z_{p^{2}-2}$ (for that odd prime $p$ for which $p^{2}-2$ is a prime number). Let $G=\Gamma\left(R_{3}\right)$ be the zero-divisor graph with vertex set $V=\mathrm{Z}\left(R_{3}\right)^{*}$. Since, $G=\Gamma\left(R_{3}\right)$ is connected [1], we have $n_{G}(V)=\left|N_{G}(V)\right|=|V|=\left|Z\left(R_{3}\right)^{*}\right|$. But from Theorem 4.1, we have $\left|Z\left(R_{3}\right)^{*}\right|=p^{2}+p-4$. Therefore, $n_{G}(V)=p^{2}+p-4$. This implies $n_{G}(V)=\left(p^{2}-3\right)+(p-1)$. Also, $\Delta(G)=p^{2}-3$ and $\delta(G)=p-1\left[\right.$ from Theorem 4.1]. This gives $n_{G}(V)=\Delta(G)+$ $\delta(G)$.

Remark: If $p=2$, then $R_{3}=Z_{2} \times Z_{2}$. In this case $V=\mathrm{Z}\left(R_{3}\right)^{*}=$ $\{(0,1),(1,0)\}$ and $G=\Gamma\left(R_{3}\right)$ is a $1-$ regular graph. Also, $n_{G}(V)$ $=2=2 \Delta(G)=2 \delta(G)$.

\section{DEFINITIONS AND RELATIONS:}

Let $R$ be a commutative ring with unity and let $a \in R$. Then annihilator of $a$ is denoted by $\operatorname{ann}(a)$ and defined by $\operatorname{ann}(a)=\{x \in R: a x=0\}$. Let $a n n *(a)=\{x(\neq 0) \in R: a x=0\}$.

The degree of a vertex $v$ of a graph $G$ denoted by $\operatorname{deg}(v)$ is the number of lines incident with $v$.

Given a zero-divisor graph $\Gamma(R)$ with vertex set $\mathrm{Z}(R)^{*}$, then degree of a vertex $v$ of $\Gamma(R)$ is given by $\operatorname{deg}(v)=$ $|a n n *(v)|$.

Let $A$ and $B$ be two commutative rings with unity. Then the direct product $A \times B$ of $A$ and $B$ is also a commutative ring with unity.

Let $G$ be a graph and $V(G)$ be the vertex set of $G$. Let $a, b \in V(G)$. We define a relation $\mathscr{R}$ on $V(G)$ as follows. For $a, b \in V(G), a$ is related to $b$ under the relation $\mathscr{R}$ if and only if $a$ and $b$ are not adjacent and for any $x \in V(G), \quad a$ and $x$ are adjacent if and only if $b$ and $x$ are adjacent. We denote this relation by $a \Re b$.

\section{Results of annihilators on $\Gamma(A \times B)$ :}

Theorem 6.1: The relation $\mathscr{R}$ is an equivalence relation on $V(G)$, where $G$ is any graph.

Proof: For every $a \in V(G)$, we have $a \Re a$, as $G$ has no selfloop. For $a, b \in V(G), a \Re b$, then clearly, $b \mathfrak{R} a$. Again let 
$a \Re b$ and $b R c$. If possible suppose, $a$ and $c$ are adjacent. Then we have $b$ and $c$ are also adjacent, a contradiction. So, $a$ and $c$ are not adjacent. Also for $x \in V(G), a$ and $x$ are adjacent $\Leftrightarrow b$ and $x$ are adjacent $\Leftrightarrow c$ and $x$ are adjacent. Therefore, $a \mathfrak{R} c$. Hence, the relation $\mathscr{R}$ is an equivalence relation on $V(G)$.

Theorem 6.2: For distinct $a, b \in Z(A \times B)^{*}, a \mathcal{R} b$ in $\Gamma(A \times B)$ if and only if $\operatorname{ann}(a)-\{a\}=\operatorname{ann}(b)-\{b\}$. Moreover, if $a \mathfrak{R} b$ in $\Gamma\left(R_{1} \times R_{2}\right)$, then $\operatorname{ann}\left(a_{1}\right)-\left\{a_{1}\right\}=\operatorname{ann}\left(b_{1}\right)-\left\{b_{1}\right\}$ and $\operatorname{ann}\left(a_{2}\right)-\left\{a_{2}\right\}=\operatorname{ann}\left(b_{2}\right)-\left\{b_{2}\right\}$, where $a=\left(a_{1}, a_{2}\right), b=$ $\left(b_{1}, b_{2}\right), a_{1}, a_{2} \in A$ and $b_{1}, b_{2} \in B$.

Proof: First suppose, for distinct $a, b \in \mathrm{Z}(A \times B)^{*}, a \mathfrak{R} b$ in $\Gamma(A \times B)$. Let $x \in \operatorname{ann}(a)-\{a\}$. This gives $a x=0, a \neq x$. So, $a$ and $x$ are adjacent. Since $a \mathfrak{R} b$ we have $b$ and $x$ are adjacent. Therefore, we have $b x=0, b \neq x$. Hence, $x \in \operatorname{ann}(b)-\{b\}$. This implies ann $(a)-\{a\} \subseteq \operatorname{ann}(b)-\{b\}$. Similarly, ann $(b)-$ $\{b\} \subseteq \operatorname{ann}(a)-\{a\}$. This gives $\operatorname{ann}(a)-\{a\}=\operatorname{ann}(b)-\{b\}$.

Conversely suppose, $\operatorname{ann}(a)-\{a\}=\operatorname{ann}(b)-\{b\}$. Assume that $a$ and $b$ are adjacent. This gives $a b=0 \Rightarrow$ $b \in \operatorname{ann}(a)-\{a\}=\operatorname{ann}(b)-\{b\}$, a contradiction. So, $a$ and $b$ are not adjacent. Again for $x \in \mathrm{Z}(A \times B)^{*}, a$ and $x$ are adjacent $\Leftrightarrow \quad a x=0 \Leftrightarrow x \in \operatorname{ann}(b)-\{b\} \Leftrightarrow b x=0 \Leftrightarrow b$ and $x$ are adjacent. This gives $a \Re b$ in $\Gamma(A \times B)$.

If $a=\left(a_{1}, a_{2}\right), b=\left(b_{1}, b_{2}\right) \in \mathrm{Z}(A \times B)^{*}$, let $x \in \operatorname{ann}(a)$ $-\{a\}$, where $x=\left(x_{1}, x_{2}\right)$. Then $a x=0, a \neq x$. Therefore, $a$ and $x$ are adjacent. Since $a \mathfrak{R} b$ in $\Gamma(A \times B)$, we have $a$ and $x$ are adjacent $\Leftrightarrow b$ and $x$ are adjacent. So, $a x=0 \Leftrightarrow b x=0$. This gives $a_{1} x_{1}=0, a_{2} x_{2}=0 \Leftrightarrow b_{1} x_{1}=0, b_{2} x_{2}=0$. Hence we have $a_{l} x_{1}=0 \Leftrightarrow b_{1} x_{1}=0,\left(x_{1} \neq a_{1}, b_{1}\right)$ in $A$ and $a_{2} x_{2}=0 \Leftrightarrow b_{2} x_{2}=0$ $\left(x_{2} \neq a_{2}, b_{2}\right)$ in $B$. Therefore, $\operatorname{ann}\left(a_{1}\right)-\left\{a_{1}\right\}=\operatorname{ann}\left(b_{1}\right)-\left\{b_{1}\right\}$ and $\operatorname{ann}\left(a_{2}\right)-\left\{a_{2}\right\}=\operatorname{ann}\left(b_{2}\right)-\left\{b_{2}\right\}$.

Example 6.3: Consider the commutative ring $Z_{2} \times Z_{4}=\{(0,0)$, $(0,1),(0,2),(0,3),(1,0),(1,1),(1,2),(1,3)\}$ and zero-divisor graph $\Gamma\left(Z_{2} \times Z_{4}\right)$. Here $Z\left(Z_{2} \times Z_{4}\right)^{*}=\{(0,1),(0,2),(0,3),(1,0)$, $(1,2)\}$.The possible edges are $\{(0,1),(1,0)\},\{(0,2),(1,0)\}$, $\{(0,3),(1,0)\}$ and $\{(0,2),(1,2)\}$. The pairs $\{(0,1),(0,2)\}$, $\{(0,1),(0,3)\},\{(0,2),(0,3)\}$ and $\{(1,0),(1,2)\}$ establish the existence of relation $\mathcal{R}$ and Theorem 6.1:

\section{CONCLUSIONS:}

In this paper, we study the adjacency matrix and neighborhood associated with zero-divisor graph for direct product of finite commutative rings. Neighborhoods may be used to represent graphs in computer algorithms, via the adjacency list and adjacency matrix representations. Neighborhoods are also used in the clustering coefficient of a graph, which is a measure of the average density of its neighborhoods. In addition, many important classes of graphs may be defined by properties of their neighborhoods.

\section{REFERENCES:}

[1] Anderson, D. F., Livingston, P.S. 'The zero-divisor graph of a commutative ring', Journal of Algebra 217 (1999), $434-447$.

[2] Beck, I. 'Coloring of commutative rings', J. Algebra 116 (1988), 208-226.
[3] DeMeyer, F. R., Mckenzie, T., Schneider, K. 'The Zerodivisor Graph of a Commutative Semi-groups', Semi Group Forum 65, (2002), 206-214.

[4] Duane, A. 'Proper Coloring and p-partite Structures of the Zero-divisor Graph', Rose Hulman Undergraduate Math Journal (2006).

[5] Harary, F. 'Graph Theory' Addison-Wesley Publishing Company, Inc (1969).

[6] Khanna, V. K., Bhambri, S. K. 'A Course in Abstract Algebra', VIKAS Publishing House Pvt. Ltd. (1993).

[7] Kulli, V. R., Sigarkanti, S. C. 'Further result on the neighbourhood number of a graph', Indian J. pure appl. Math., 23(8) (1992), 575-577.

[8] MATHEMATICA, Wolfram Software.

[9] Redmond, S. P. 'The zero-divisor graph of a noncommutative ring', International J. Commutative Rings 1(4) (2002), $203-211$.

[10] Sharma, P., Sharma, A., Vats, R. K. 'Analysis of Adjacency Matrix and Neighborhood Associated with Zerodivisor Graph of Finite Commutative Rings', International Journal of Computer Applications, Volume 14 No.3 (2011), 0975-8887. 